\title{
Radiotherapy versus Surgery in Early-Stage HPV-Positive Oropharyngeal Cancer
}

\author{
Dong-Yun Kim ${ }^{1}$, Hong-Gyun Wu', Jin Ho Kim ${ }^{1}$, Joo Ho Lee', Soon-Hyun Ahn'², Eun-Jae Chung' ${ }^{2}$ Keun-Yong Eom ${ }^{3}$, Young Ho Jung ${ }^{4}$, \\ Woo-Jin Jeong ${ }^{4}$, Tack-Kyun Kwon ${ }^{5}$, Suzy Kim ${ }^{6}$, Chan Woo Wee ${ }^{6}$ \\ Departments of ${ }^{1}$ Radiation Oncology and ${ }^{2}$ Otorhinolaryngology-Head and Neck Surgery, Seoul National University Hospital, Seoul, Departments of \\ ${ }^{3}$ Radiation Oncology and ${ }^{4}$ Otorhinolaryngology-Head and Neck Surgery, Seoul National University Bundang Hospital, Seongnam, Departments of \\ ${ }^{5}$ Otorhinolaryngology-Head and Neck Surgery and ${ }^{6}$ Radiation Oncology, SMG-SNU Boramae Medical Center, Seoul, Korea
}

Purpose This study aimed to compare the outcomes of primary radiotherapy (RT) versus surgery in early-stage human papilloma virus-positive oropharyngeal squamous cell carcinoma (hpv+OPC), and investigate the preoperative clinical factors that can predict the requirement for postoperative adjuvant treatment.

Materials and Methods This multicenter study included 166 patients with American Joint Committee on Cancer 8th edition-Stages I-II hpv+OPC. Sixty (36.1\%) and 106 (63.9\%) patients underwent primary (concurrent chemo)radiotherapy [(CC)RT] and surgery, respectively. Seventy-eight patients $(73.6 \%)$ in the surgery group received postoperative (CC)RT.

Results With a median follow-up of 45.6 months for survivors, the 2-year overall survival (OS), progression-free survival (PFS), and locoregional control (LC) for RT/surgery were 97.8\%/96.4\%, 91.1\%/92.0\%, and 92.9\%/93.3\%, respectively. In multivariate analyses, patients with synchronous radiologic extranodal extension and conglomeration (ENEcong) of metastatic lymph nodes (LNs) showed significantly poorer OS ( $p=0.047)$, PFS $(p=0.001)$, and LC $(p=0.003)$. In patients undergoing primary surgery, two or more clinically positive LN metastases (odds ratio [OR], 5.15; $p=0.004$ ) and LN metastases with ENEcong (OR, 3.75; $p=0.009)$ were predictors of postoperative chemoradiotherapy. No patient in the primary RT group demonstrated late severe toxicity whereas three (2.8\%), one (0.9\%), and one (0.9\%) patient in the surgery group showed grade 3 dysphagia, grade 3 xerostomia, and fatal oral cavity bleeding.

Conclusion We found no differences in OS, PFS, and LC between upfront RT and surgery in stage I-II hpv+OPC which warrants comparison through a prospective trial in the treatment de-escalation era. However, most early-stage hpv+OPC patients undergoing surgery received adjuvant (CC)RT. Pretreatment LN findings were prognostic and predictive for adjuvant treatment.

Key words HPV-positive oropharyngeal cancer, Oropharynx, HPV, Radiotherapy, General surgery

\section{Introduction}

The incidence of oropharyngeal squamous cell carcinoma (OPC) caused by human papillomavirus (HPV) infection continues to increase worldwide [1]. HPV-positive OPC (hpv+OPC) is known to be more radiosensitive [2] and has a better overall prognosis than HPV-unrelated OPC [3]. The 5-year overall survival (OS) of hpv+OPC in the 8th edition of the American Joint Committee on Cancer (AJCC) staging was reported to be as high as $93.9 \%$ [3]. In addition, hpv+OPC mostly occurs in relatively younger, non-smoking, and non-drinking patients than in HPV-unrelated OPC [4]. For these reasons, hpv+OPC has been separately staged from HPV-negative OPC ever since the 8th edition of AJCC was published [3,5]. Considering the good prognosis and young age at diagnosis [6], reducing the occurrence of late toxicity is a priority in patients diagnosed with hpv+OPC.

The standard management of early-stage (stage I, II by AJCC 8th edition) OPC includes either definitive radiothera- py (RT) or surgery. Definitive RT as a primary treatment was historically one of the first-line choices for early-stage OPC [4]. Adding concurrent chemotherapy to primary RT (concurrent chemoradiotherapy [CCRT]) is recommended for cases of multiple lymph node (LN) metastases, a single LN metastasis larger than $3 \mathrm{~cm}$, or those having adverse features such as extranodal extension (ENE) [7]. Minimally invasive surgical techniques such as transoral robotic surgery (TORS) or transoral laser microsurgery (TLM) are gaining popularity as the primary treatment for OPC [8]. However, RT techniques such as intensity-modulated radiation therapy using simultaneous integrated boost (SIB-IMRT) have also dramatically evolved to deliver conformal dose distributions with better normal tissue sparing. In a recent phase II randomized controlled trial (RCT), the "ORATOR" study, patients diagnosed with T1-2, N0-2, M0 were randomly assigned to primary (concurrent chemo)radiotherapy [(CC)RT] or TORS. There was no difference in OS and progression-free survival (PFS) in both treatment groups and the two treatment meth-

Correspondence: Chan Woo Wee

Department of Radiation Oncology, SMG-SNU Boramae Medical Center, 20, Boramae-ro 5-gil, Dongjak-gu, Seoul 07061, Korea

Tel: 82-2-870-1695 Fax: 82-2-870-1689 E-mail: wcw0108@hanmail.net

Received April 7, 2021 Accepted June 22, 2021 Published Online June 23, 2021 
ods showed different toxicity profiles [9]. In addition, there have been many studies on de-intensification of treatment considering the favorable prognosis of hpv+OPC [10], but there are no actual differences in treatment methods according to p16 or HPV status.

After primary surgery, adjuvant therapy is determined based on pathologic findings. Generally, adjuvant RT is recommended if the patient shows positive/ close resection margins, pathologic ENE (pENE), pT3-T4 disease, multiple LN metastases, perineural invasion, or lymphovascular invasion [11]. Concurrent cisplatin-based chemotherapy is also recommended for patients with pENE or positive margins [12]. In such cases, bimodal or trimodal treatment may negatively affect treatment-related long-term sequelae [13]. Therefore, it is important to decide the first-line treatment (RT vs. surgery) based on preoperative clinical factors to reduce the number of treatment modalities delivered.

In this study, we compared the clinical outcomes and toxicity of primary (CC)RT and surgery in early-stage hpv+OPC as well as investigated the preoperative clinical factors that can predict the requirement for postoperative (CC)RT.

\section{Materials and Methods}

\section{Study design and patients}

We analyzed 166 patients diagnosed with early-stage hpv+OPC between January 2006 and December 2019. The eligibility criteria were as follows: (1) age 18 years or older, (2) histologically proven p16 and/or hpv+OPC, (3) stage I-II by AJCC 8th edition, (4) primary RT or surgery with curative intent, and (5) Eastern Cooperative Oncology Group performance scale $0-1$. Since expression of p16 is considered a surrogate for HPV infection, all patients in this study had positive results on p16-immunohistochemical staining. We excluded patients who received induction chemotherapy or cetuximab-based CCRT, which was proven inferior to cisplatin-based CCRT in the DE-ESCALaTE HPV and Radiation Therapy Oncology Group 1016 trial [7,14]. Patients with a single LN metastasis $>3 \mathrm{~cm}$ or multiple LN metastases treated with RT but without chemotherapy were also excluded from the analysis. All patients were evaluated by a multidisciplinary head and neck tumor board. Treatment options were decided after comprehensive evaluation by physical examination and imaging modalities such as magnetic resonance imaging (MRI), ${ }^{18} \mathrm{~F}$-fluorodeoxyglucose positron emission tomography $\left({ }^{18} \mathrm{~F}-\mathrm{FDG}-\mathrm{PET}\right)$, or computed tomography (CT).

\section{Definition of clinically metastatic LN and clinical ENE}

Clinically metastatic LNs were evaluated on pretreatment MRI/CT scans with or without ${ }^{18} \mathrm{~F}-\mathrm{FDG}$-PET. Of all patients, 95.8\% underwent ${ }^{18} \mathrm{~F}-\mathrm{FDG}$-PET. An LN was defined as a metastatic LN when it met as least one of the following four criteria: (1) ${ }^{18} \mathrm{~F}-\mathrm{FDG}$-PET-ovid, maximum standardized uptake value $\geq 2.5$ [15]; (2) long diameter $>15 \mathrm{~mm}$ and short diameter > $10 \mathrm{~mm}$ on MRI/CT; (3) sonography (SONO)-guided fine-needle aspiration or SONO-guided biopsy-positive; or (4) necrotic [16-19].

Clinical ENE of the metastatic LN (cENE) was defined as expansion of tumor cells beyond the LN capsule into the perinodal adipose tissue which showed enhancement, thickening, and irregularity of the nodal rim or infiltration into the adjacent fat or other soft tissue planes [17]. The criteria for conglomeration of the metastatic LNs were juxtaposition of two or more LNs abutting one another with loss of intervening fat plane $[20,21]$. When metastatic LNs represented both cENE and conglomeration, the patient was considered
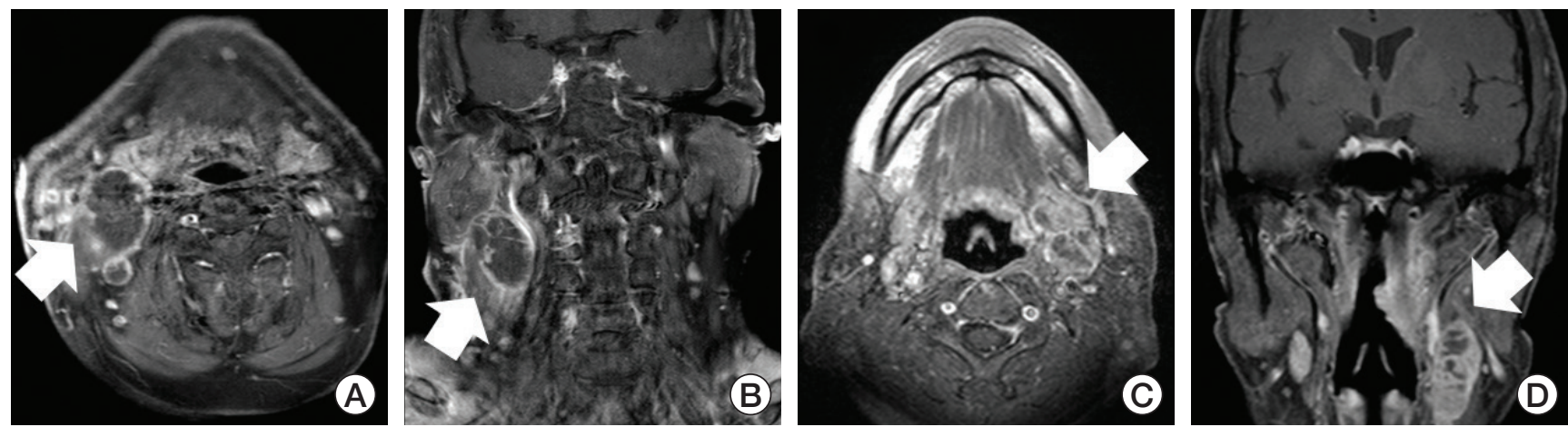

Fig. 1. Representative cases of clinical extranodal extention of the metastatic lymph node (LN) (cENE) and metastatic lymph nodes with cENE and conglomeration (ENEcong) in magnetic resonance imaging (arrow) on axial view $(\mathrm{A}, \mathrm{C})$ and coronal view (B, D). ENEcong was defined as two or more metastatic LNs abutting one another with loss of intervening fat plane accompanying tumor cells beyond the LN capsule into the perinodal adipose tissue. 
Table 1. Patient characteristics and comparison between primary radiotherapy vs. surgery arms

\begin{tabular}{|c|c|c|c|c|}
\hline Characteristic & Total $(n=166)$ & RT $(n=60)$ & Surgery $(n=106)$ & p-value ${ }^{a}$ \\
\hline Age (yr) & $58.5(36-86)$ & $59(46-77)$ & $58(36-86)$ & $0.559^{b)}$ \\
\hline \multicolumn{5}{|l|}{ Age (yr) } \\
\hline$<60$ & $93(56.0)$ & $32(53.3)$ & $61(57.5)$ & 0.599 \\
\hline$\geq 60$ & $73(44.0)$ & $28(46.7)$ & $45(42.5)$ & \\
\hline \multicolumn{5}{|l|}{ Sex } \\
\hline Male & $139(83.7)$ & $48(80.0)$ & $91(85.8)$ & 0.327 \\
\hline Female & $27(16.3)$ & $12(20.0)$ & $15(14.2)$ & \\
\hline \multicolumn{5}{|l|}{ Primary site } \\
\hline Tonsil & $143(86.1)$ & $50(83.3)$ & $93(87.7)$ & 0.678 \\
\hline Others ${ }^{c}$ & $23(13.9)$ & $10(16.7)$ & $13(12.3)$ & \\
\hline \multicolumn{5}{|l|}{ ACCI } \\
\hline $1-5$ & 157 (94.6) & $56(93.3)$ & $101(95.3)$ & 0.594 \\
\hline $6-9$ & $9(5.4)$ & $4(6.7)$ & $5(4.7)$ & \\
\hline \multicolumn{5}{|l|}{ Smoking history } \\
\hline$\leq 10$ pack-years & $77(46.4)$ & $36(60.0)$ & $41(38.7)$ & 0.008 \\
\hline > 10 pack-years & $89(53.6)$ & $24(40.0)$ & $65(61.3)$ & \\
\hline \multicolumn{5}{|l|}{ Clinical T category } \\
\hline T0-T1 & $44(26.5)$ & $6(10.0)$ & $38(35.8)$ & $<0.001$ \\
\hline T2-T3 & $122(73.5)$ & $54(90.0)$ & $68(64.2)$ & \\
\hline \multicolumn{5}{|l|}{ Clinical N category } \\
\hline N0 & $17(10.2)$ & $3(5.0)$ & $14(13.2)$ & 0.003 \\
\hline N1 & $132(79.5)$ & $45(75.0)$ & $87(82.1)$ & \\
\hline N2 & $17(10.2)$ & $12(20.0)$ & $5(4.7)$ & \\
\hline \multicolumn{5}{|c|}{ Clinical stage (AJCC 8th) } \\
\hline I & $144(86.7)$ & $47(78.3)$ & $97(91.5)$ & 0.016 \\
\hline II & $22(13.3)$ & $13(21.7)$ & $9(8.5)$ & \\
\hline \multicolumn{5}{|c|}{ Metastatic LN with clinical extranodal extension } \\
\hline No & $91(54.8)$ & $32(53.3)$ & $59(55.7)$ & 0.265 \\
\hline Yes & $75(45.2)$ & $28(46.7)$ & $47(44.3)$ & \\
\hline
\end{tabular}

Values are presented as median (range) or number (\%). ACCI, age-adjusted Charlson comorbidity index; AJCC, American Joint Committee on Cancer; LN, lymph node; RT, radiotherapy. ${ }^{a}$ Chi-square test, ${ }^{b}$ Independent $t$ test, ${ }^{c}$ Others: Base of tongue, soft palate, posterior pharyngeal wall, vallecular.

to have clinical "ENEcong" [21] (Fig. 1).

\section{Primary RT}

Sixty patients $(36.1 \%)$ were treated with primary RT. RT was administered using IMRT ( $\mathrm{n}=52)$ or 3-dimensional conformal $\mathrm{RT}(\mathrm{n}=8)$. The most frequently used RT regimen was $67.5 \mathrm{~Gy}$ in 30 fractions over 6 weeks using the SIB-IMRT technique. The median total dose was $67.5 \mathrm{~Gy}$ (range, 54 to 72 Gy) and the median fraction number was 30 (range, 27 to 35). The prescribed doses for high-risk, subclinical intermediaterisk, and low-risk areas were 67.5-70, 54-56, and 42-47.6 Gy, respectively. Bilateral neck irradiation was administered to $85.0 \%$ of patients treated with primary RT.

CCRT was administrated to most patients $(96.7 \%)$. Of those, weekly $\left(35 \mathrm{mg} / \mathrm{m}^{2}\right)$ or triweekly $\left(100 \mathrm{mg} / \mathrm{m}^{2}\right)$ intrave- nous cisplatin was delivered in $91.4 \%$ and $8.6 \%$ of patients, respectively.

\section{Primary surgery}

One-hundred six patients $(63.9 \%)$ underwent primary surgery. Of the patients treated with primary surgery, $35.8 \%$ $(n=38)$ and $64.2 \% \quad(n=68)$ underwent TORS and oropharyngectomy, respectively. Neck LN dissection was performed in $94.3 \%$ of patients. The median number of dissected LNs was 35 (range, 0 to 77), while that of positive LNs was 2 (range, 0 to 17). The median maximal size of the pathologically dissected LNs was $18 \mathrm{~mm}$ (range, 0 to $67 \mathrm{~mm}$ ). Forty-two patients $(39.6 \%)$ showed pENE. Lymphovascular invasion was found in 33 patients $(31.1 \%)$. Resection margin was reported as 'clear (> $1 \mathrm{~mm})$ ', 'close (0-1 mm)', and 'involved' 
A

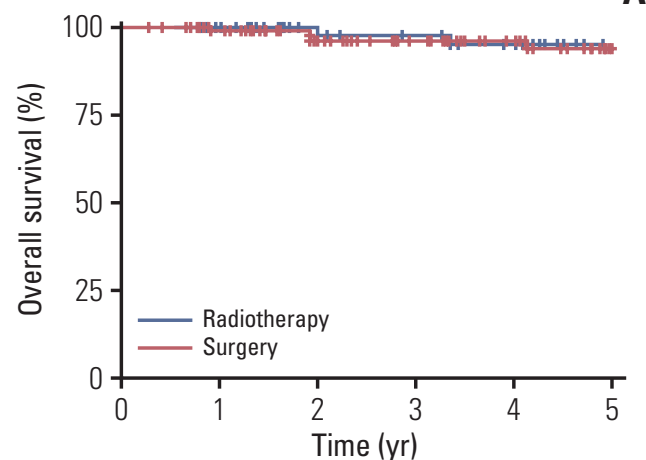

No. at risk

$\begin{array}{rrrrrrr}\text { Radiotherapy } & 60 & 57 & 44 & 39 & 35 & 23 \\ \text { Surgery } 106 & 92 & 75 & 55 & 44 & 23\end{array}$

B

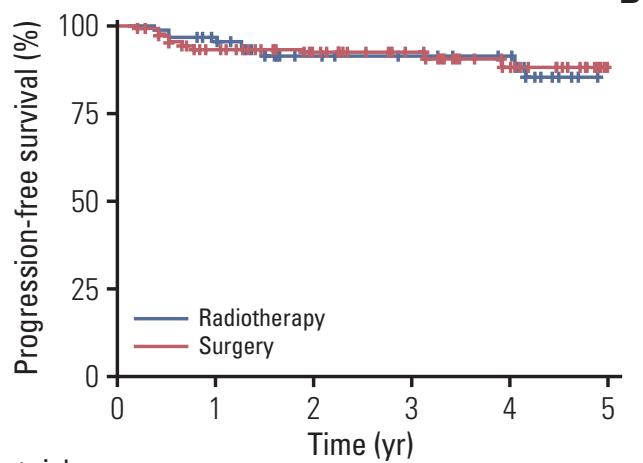

No. at risk

$\begin{array}{rrrrrrr}\text { Radiotherapy } & 60 & 55 & 41 & 36 & 33 & 20 \\ \text { Surgery } & 106 & 88 & 72 & 54 & 42 & 22\end{array}$

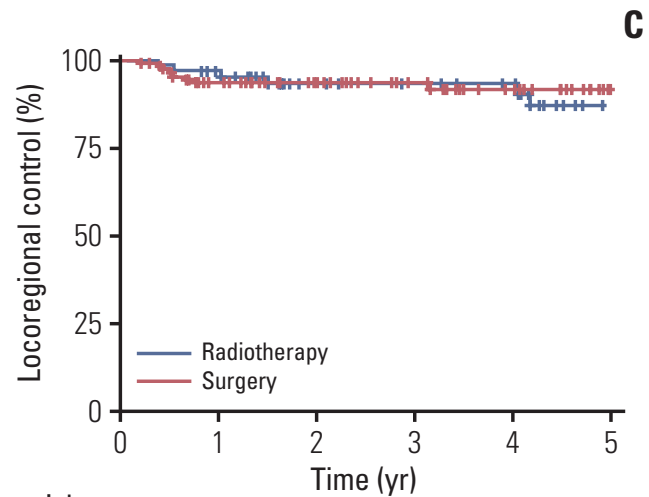

No. at risk

$\begin{array}{rrrrrrr}\text { Radiotherapy } & 60 & 55 & 41 & 36 & 33 & 20 \\ \text { Surgery } & 106 & 88 & 72 & 54 & 43 & 22\end{array}$

Fig. 2. Overall survival $(\mathrm{p}=0.755)(\mathrm{A})$, progression-free survival $(\mathrm{p}=0.810)(\mathrm{B})$, and locoregional control $(\mathrm{p}=0.721)(\mathrm{C})$ curves of patients treated by (chemo)radiotherapy vs. surgery-based treatment ( $\mathrm{p}$-value by log-rank test). in $68.0 \%, 31.1 \%$, and $0.9 \%$ of patients, respectively. Of all, 78 patients had adjuvant therapy and 28 didn't have any adjuvant treatment. Those of all 78 patients received adjuvant RT. Of them, 39 patients had postoperative CCRT and 39 did only postoperative RT.

Those of 78 patients $(73.6 \%)$ required postoperative RT, while 39 patients $(36.8 \%)$ received postoperative CCRT. The median total dose of adjuvant RT was $63 \mathrm{~Gy}$ (range, 55 to 70 Gy) and the prescribed doses were 60-67.5, 52-56, and 42-47.6 Gy for high-risk, intermediate-risk, and low-risk regions, respectively. Of the 78 patients who received adjuvant RT, 54 received bilateral neck irradiation. Weekly intravenous cisplatin $\left(35 \mathrm{mg} / \mathrm{m}^{2}\right)$ was administered as adjuvant CCRT.

\section{Follow-up}

After completion of treatment, clinical assessment was performed every 1-3 months during the first year of followup, every 3-4 months during the second year, and every 6 months from the third year. Follow-up imaging was performed at least every 6 months. Late toxicity was considered a side effect that persisted beyond post-treatment 6 months. Toxicity was graded using the Common Terminology Criteria for Adverse Events ver. 5.0.

\section{Statistical analysis}

Patient characteristics between the primary RT and surgery groups were compared using an independent $t$ test for age, the number of clinically positive LNs, and maximum size of clinically positive LNs. Other demographic variables between the two treatment groups were compared using the chi-square test. We compared the clinical outcomes such as OS, PFS, and locoregional control (LC) between the two treatments using the log-rank test. OS, PFS, and LC rates were calculated using the Kaplan-Meier method. Clinical factors associated with OS, PFS, and LC were evaluated using univariate and multivariate analyses with the backward stepwise Cox regression model. A mixed-effects logistic regression analysis was used to identify factors that affected clinical outcomes and predict the need for postoperative RT and CCRT. All statistical analyses were two-sided and performed using Stata/MP 15.0 (StataCorp, College Station, $T X)$, with a significance level of $<0.05$.

\section{Results}

A total of 166 patients diagnosed with early-stage (AJCC 8th stage I-II) hpv+OPC were analyzed. The median followup was 45.6 months for survivors (range, 3.5 to 149.3 months). We compared the outcomes according to primary treatment modality (RT vs. surgery). Among these 166 patients, 60 


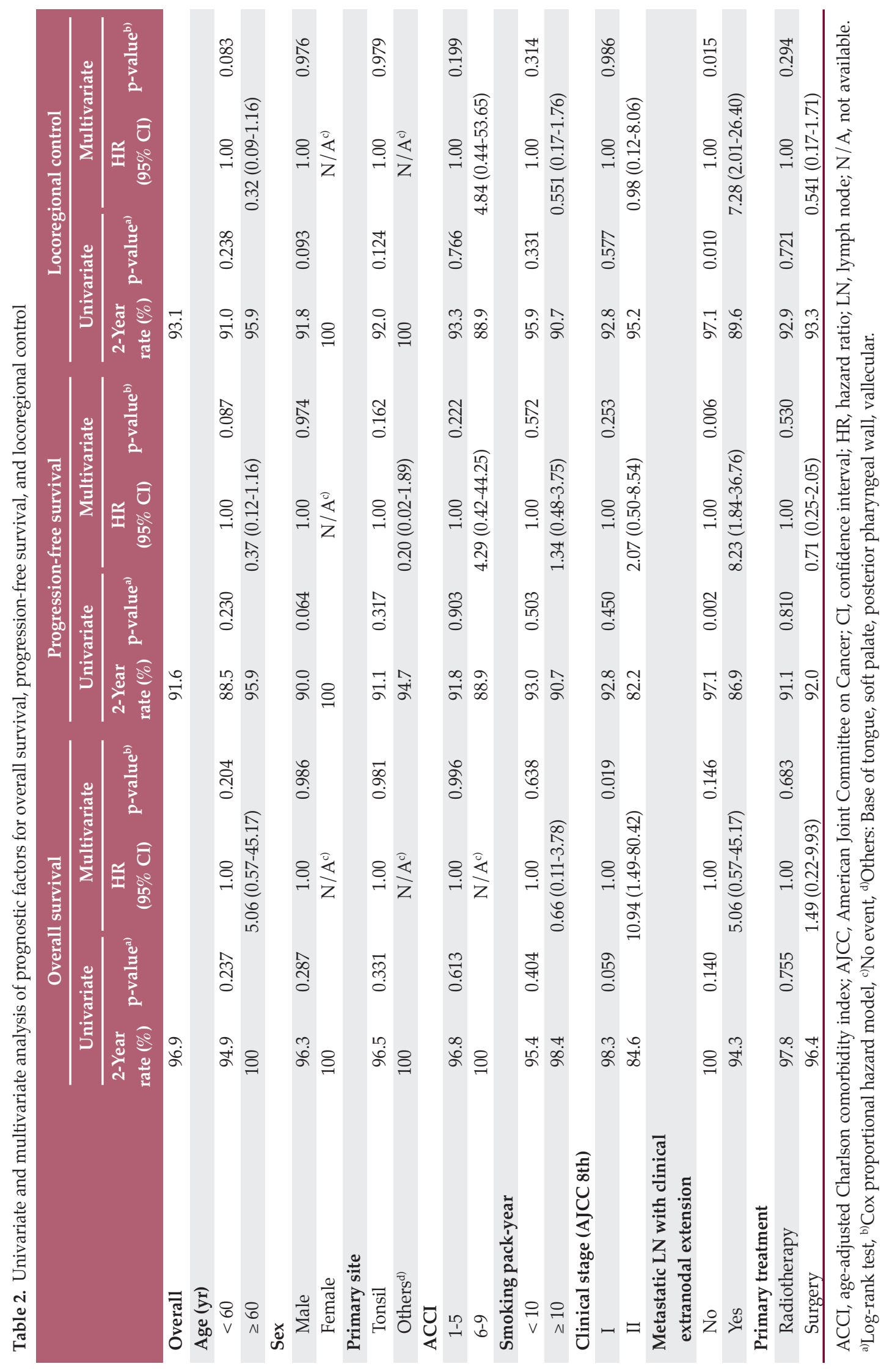


Table 3. Multivariate analyses showing the prognostic significance of metastatic lymph nodes with extranodal extension and conglomeration

\begin{tabular}{|c|c|c|c|c|c|c|}
\hline \multirow{2}{*}{ Variable } & \multicolumn{2}{|c|}{ Overall survival } & \multicolumn{2}{|c|}{ Progression-free survival } & \multicolumn{2}{|c|}{ Locoregional control } \\
\hline & HR $(95 \%$ CI) & p-value $\left.{ }^{a}\right)$ & HR $(95 \% \mathrm{CI})$ & p-value ${ }^{a}$ & HR $(95 \% \mathrm{CI})$ & p-value \\
\hline \multicolumn{7}{|l|}{ Age (yr) } \\
\hline$<60$ & 1.00 & 0.375 & 1.00 & 0.156 & 1.00 & 0.144 \\
\hline$\geq 60$ & $2.70(0.30-24.16)$ & & $2.30(0.73-7.29)$ & & $2.64(0.72-9.75)$ & \\
\hline \multicolumn{7}{|l|}{ Sex } \\
\hline Male & 1.00 & 0.981 & 1.00 & 0.975 & 1.00 & 0.977 \\
\hline Female & $\mathrm{N} / \mathrm{A}^{\mathrm{b})}(\mathrm{N} / \mathrm{A})$ & & $\mathrm{N} / \mathrm{A}^{\mathrm{b})}(\mathrm{N} / \mathrm{A})$ & & $\mathrm{N} / \mathrm{A}^{\mathrm{b})}(\mathrm{N} / \mathrm{A})$ & \\
\hline \multicolumn{7}{|l|}{ Primary site } \\
\hline Tonsil & 1.00 & 0.972 & 1.00 & 0.163 & 1.00 & 0.980 \\
\hline Others ${ }^{c)}$ & $\mathrm{N} / \mathrm{A}^{\mathrm{b})}(\mathrm{N} / \mathrm{A})$ & & $0.21(0.02-1.88)$ & & $\mathrm{N} / \mathrm{A}^{\mathrm{b})}(\mathrm{N} / \mathrm{A})$ & \\
\hline \multicolumn{7}{|l|}{ ACCI } \\
\hline $1-5$ & 1.00 & 0.993 & 1.00 & 0.353 & 1.00 & 0.315 \\
\hline $6-9$ & $\mathrm{~N} / \mathrm{A}^{\mathrm{b})}(\mathrm{N} / \mathrm{A})$ & & $2.97(0.30-29.57)$ & & $3.34(0.32-35.10)$ & \\
\hline \multicolumn{7}{|l|}{ Smoking history } \\
\hline$\leq 10$ pack-years & 1.00 & 0.639 & 1.00 & 0.618 & 1.00 & 0.393 \\
\hline$>10$ pack-years & $1.52(0.27-8.65)$ & & $1.30(0.46-3.64)$ & & $1.67(0.52-5.37)$ & \\
\hline \multicolumn{7}{|c|}{ Clinical stage (AJCC 8th) } \\
\hline $\mathrm{I}$ & 1.00 & 0.018 & 1.00 & 0.316 & 1.00 & 0.875 \\
\hline II & $13.21(1.55-112.63)$ & & $2.07(0.50-8.54)$ & & $0.84(0.10-7.01)$ & \\
\hline \multicolumn{7}{|c|}{$\begin{array}{l}\text { Metastatic LN with clinical } \\
\text { extranodal extension and } \\
\text { conglomeration }\end{array}$} \\
\hline No & 1.00 & 0.047 & 1.00 & 0.001 & 1.00 & 0.003 \\
\hline Yes & $9.77(1.03-92.50)$ & & $9.18(2.62-32.12)$ & & $7.28(2.01-26.40)$ & \\
\hline \multicolumn{7}{|l|}{ Primary treatment } \\
\hline Radiotherapy & 1.00 & 0.460 & 1.00 & 0.709 & 1.00 & 0.433 \\
\hline Surgery & $2.12(0.29-15.44)$ & & $0.82(0.28-2.36)$ & & $0.63(0.19-2.02)$ & \\
\hline
\end{tabular}

AJCC, American Joint Committee on Cancer; CI, confidence interval; HR, hazard ratio; LN, lymph node; N/A, not available. ${ }^{a}$ Cox proportional hazard model, ${ }^{b}$ No event, ${ }^{c}$ Others: Base of tongue, soft palate, posterior pharyngeal wall, vallecular.

(36.1\%) were treated with primary (CC)RT, while $106(63.9 \%)$ patients underwent primary surgery. The median ages of the primary RT and surgery groups were 59 years (range, 46 to 77 years) and 58 years (range, 36 to 86 years), respectively. The clinical demographics of the patients are presented in Table 1. There was no difference in sex, site of primary disease, and age-adjusted Charlson comorbidity index between the RT and surgery groups. Twenty-four (60.0\%) and $41(38.7 \%)$ patients had a smoking history of 10 pack-years or more in the RT and surgery arms, respectively $(\mathrm{p}=0.008)$. Patients treated with RT had higher clinical stage $(\mathrm{p}=0.016)$, T category $(\mathrm{p}<0.001)$, and $\mathrm{N}$ category $(\mathrm{p}=0.003)$ than those in the surgery group. Meanwhile, no significant difference was found in cENE between the two treatment arms $(\mathrm{p}=0.265)$.

There was no difference in OS, PFS, and LC between the RT and surgery arms (Fig. 2). The 2-year OS, PFS, and LC rates for the (CC)RT/surgery arms were $97.8 \% / 96.4 \%(\mathrm{p}=0.755)$,
91.1\% / 92.0\% ( $\mathrm{p}=0.810)$, and 92.9\% /93.3\% ( $\mathrm{p}=0.721)$, respectively. After adjustment for other possible confounding factors, there was no difference between the (CC)RT and surgery arms in terms of OS ( $\mathrm{p}=0.683)$, PFS $(\mathrm{p}=0.530)$, and LC $(\mathrm{p}=0.294)$ (Table 2$)$.

In multivariate analyses, cENE was a significant factor for PFS ( $\mathrm{p}=0.006)$ and LC $(\mathrm{p}=0.015)$, but no significant impact on OS was found ( $\mathrm{p}=0.146$ ) (Table 2 ). In an additional analysis, a significant difference was also found in terms of PFS and LC among the four patient groups based on the status of metastatic LNs (ENEcong vs. ENE without conglomeration vs. no ENE vs. N0 disease) (S1 Table, S2A-S2C Fig.). The 2-year PFS was $83.3 \%$ vs. $96.0 \%$ vs. $98.3 \%$ vs. $91.7 \%$ ( $\mathrm{p}=0.002$ ), and the 2-year LC was $87.0 \%$ vs. $96.0 \%$ vs. $98.3 \%$ vs. $91.7 \%$ ( $\mathrm{p}=0.012$ ), respectively. Although no significant difference was reported, the 2-year OS rates were $92.5 \%$ vs. $100.0 \%$ vs. $100 \%$ vs. $100 \%$ ( $\mathrm{p}=0.107)$, respectively. When we performed multivari- 
Table 4. Summary of late toxicity in primary radiotherapy vs. surgery arms

\begin{tabular}{|c|c|c|c|c|}
\hline \multirow{2}{*}{ Toxicity } & \multicolumn{2}{|c|}{ Radiotherapy $(n=60)$} & \multicolumn{2}{|c|}{ Surgery $(n=106)$} \\
\hline & Grade 1-2 & $\geq$ Grade 3 & Grade 1-2 & $\geq$ Grade 3 \\
\hline Aspiration & 0 & 0 & $3(2.8)$ & $1(0.9)$ \\
\hline Dysphagia & $4(6.7)$ & 0 & $10(9.4)$ & $3(2.8)$ \\
\hline Lymphedema & 0 & 0 & $4(3.8)$ & 0 \\
\hline Neck fibrosis & $2(3.3)$ & 0 & $15(14.2)$ & 0 \\
\hline Oral cavity bleeding & 0 & 0 & $1(0.9)$ & $1(0.9)$ \\
\hline Oral pain & 0 & 0 & $4(3.8)$ & 0 \\
\hline Otalgia & 0 & 0 & $2(1.9)$ & 0 \\
\hline Sensory neuropathy & $1(1.7)$ & 0 & $5(4.7)$ & 0 \\
\hline Shoulder pain & $1(1.7)$ & 0 & $16(15.1)$ & 0 \\
\hline Tinnitus & $1(1.7)$ & 0 & $4(3.8)$ & 0 \\
\hline Trismus & 0 & 0 & $3(2.8)$ & 0 \\
\hline Ulceration & 0 & 0 & $1(0.9)$ & 0 \\
\hline Xerostomia & $9(15.0)$ & 0 & $14(13.2)$ & 0 \\
\hline
\end{tabular}

Values are presented as number $(\%)$.

Table 5. Analysis of factors to predict for postoperative radiotherapy and chemoradiotherapy in patients treated with surgery

\begin{tabular}{|c|c|c|c|c|c|c|}
\hline & \multicolumn{3}{|c|}{ Postoperative RT } & \multicolumn{3}{|c|}{ Postoperative CCRT } \\
\hline & OR & $95 \% \mathrm{CI}$ & p-value ${ }^{a)}$ & OR & $95 \% \mathrm{CI}$ & p-value ${ }^{a}$ \\
\hline \multicolumn{7}{|c|}{ Clinical T category } \\
\hline $\mathrm{T} 0 / 1$ & 1.00 & & 0.765 & 1.00 & & 0.317 \\
\hline $\mathrm{T} 2 / 3$ & 1.16 & $0.43-3.11$ & & 1.66 & $0.61-4.50$ & \\
\hline \multicolumn{7}{|c|}{ Clinical N category } \\
\hline $\mathrm{N} 0 / 1$ & 1.00 & & NA & 1.00 & & 0.913 \\
\hline N2 & NA & NA & & 1.13 & $0.13-9.81$ & \\
\hline \multicolumn{7}{|c|}{ No. of clinically positive LNs } \\
\hline$<2$ & 1.00 & & 0.506 & 1.00 & & 0.004 \\
\hline$\geq 2$ & 1.55 & $0.42-5.69$ & & 5.15 & $1.68-15.74$ & \\
\hline \multicolumn{7}{|c|}{ Maximum size of clinically positive $\mathrm{LN}(\mathrm{mm})$} \\
\hline$<20$ & 1.00 & & 0.017 & 1.00 & & 0.138 \\
\hline$\geq 20$ & 4.34 & $1.31-14.45$ & & 2.61 & $0.73-9.29$ & \\
\hline \multicolumn{7}{|c|}{ Metastatic LN with clinical extranodal extension } \\
\hline No & 1.00 & & 0.650 & 1.00 & & 0.650 \\
\hline Yes & 0.72 & $0.18-2.96$ & & 0.66 & $0.18-2.96$ & \\
\hline \multicolumn{7}{|c|}{ Extranodal extension with conglomeration } \\
\hline No & 1.00 & & 0.330 & 1.00 & & 0.019 \\
\hline Yes & 1.95 & $0.51-7.52$ & & 4.83 & $1.30-17.95$ & \\
\hline
\end{tabular}

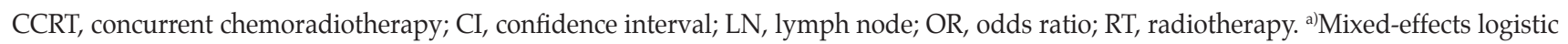
regression model.

ate analyses including ENEcong as an independent variable, patients with ENEcong had significantly poorer OS (hazard ratio [HR], 9.77; 95\% confidence interval $[\mathrm{CI}], 1.03$ to 92.51 ; $\mathrm{p}=0.047)$, PFS (HR, 9.18; 95\% CI, 2.62 to 32.12; $\mathrm{p}=0.001)$, and LC (HR, 7.28; 95\% CI, 2.01 to 26.40; $\mathrm{p}=0.003$ ) (Table 3).

None of the patients in the (CC)RT group demonstrated late grade 3 or higher toxicity, whereas three $(2.8 \%)$, one $(0.9 \%)$, and one $(0.9 \%)$ patient in the surgery group showed late grade 3 dysphagia, grade 3 aspiration, and grade 5 oral cavity bleeding, respectively (Table 4 ).

As mentioned previously, 78 patients $(73.6 \%)$ in the surgery group received postoperative RT, while 39 patients 
(36.8\%) were treated with postoperative CCRT. Multivariate analyses showed that a maximum preoperatively positive LN size of $\geq 20 \mathrm{~mm}$ (odds ratio [OR], 3.80; $\mathrm{p}=0.012$ ) was a significant factor in predicting the use of postoperative RT. Patients who had two or more clinically metastatic LN metastases (OR, 5.15; $\mathrm{p}=0.004)$ or metastatic LNs with ENEcong (OR, 3.75; $\mathrm{p}=0.009)$ showed a significantly higher possibility of receiving postoperative CCRT (Table 5). Moreover, among patients with clinical T1-2N0 disease treated with primary surgery $(n=13), 30.8 \%(4 / 13)$ received postoperative RT due to a close resection margin of $\leq 1 \mathrm{~mm}$.

\section{Discussion}

In the current study, we compared (CC)RT and surgery as initial treatments for early-stage hpv+OPC, and found no difference in OS, PFS, and LC between the two arms. Notably, $73.6 \%$ and $36.8 \%$ of patients treated with primary surgery received postoperative RT and postoperative CCRT, respectively. We found pretreatment clinical factors to predict the necessity of adjuvant therapy, but few studies have investigated this issue.

The debate on which of the two treatments is more optimal between primary (CC)RT and surgery in early-stage hpv+OPC has continued, as the two methods have reported comparable oncologic outcomes [2,8]. Like previous retrospective studies, the 'ORATOR' study, which was conducted as a phase II RCT, revealed similar OS and PFS results between the two fractionation regimens [9]. Our study showed similar results, but it still needs to be verified through ongoing prospective studies. The EORTC 1420 'Best of' trial is an ongoing phase III prospective RCT evaluating clinical outcomes and patient-reported swallowing function in T1-2 N0 OPC between novel surgical and RT strategies as well as primary TORS / TLM vs. IMRT-based RT [22]. In addition, an ongoing phase II "ORATOR 2" trial aims to compare oncologic outcomes between de-intensified primary RT and primary surgery with de-intensified adjuvant therapy [23]. Moreover, NRG-HN002 recently reported interesting results of a phase II RCT that found a suitable de-escalation treatment regimen for upcoming phase III RCT (NRG-HN005) in T1-T2 N1-N2b M0 or T3N0-N2b M0 hpv+OPC. Given the results of NRG-HN002, 60 Gy reduced-dose IMRT with weekly cisplatin showed comparable outcomes with $70 \mathrm{~Gy}$ full-dose RT with cisplatin [24]. Through these several prospective studies and de-escalation trials, an optimal treatment option may soon be determined for early-stage hpv $+\mathrm{OPC}$.

Meanwhile, it is important to note that in the current study, adjuvant therapy was administered to a significant number of patients after primary surgery in early-stage hpv+OPC.
As two-thirds of the patients received adjuvant therapy in the "ORATOR" trial $[9,23]$, our study showed that in patients treated with primary surgery, $73.6 \%$ and $36.8 \%$ received postoperative RT and postoperative CCRT, respectively. Even in clinical T1-2N0 patients, 30.8\% required postoperative RT due to close resection margins. This could be of great concern because bimodal or trimodal treatment with the addition of adjuvant RT or CCRT following primary surgery can increase acute and late toxicity, which would significantly affect patients' quality of life. Several studies have reported increasing complication rates related to adjuvant RT following primary surgery. In a multicenter study in the United States, adjuvant therapy worsened gastrostomy tube use and persistent gastrostomy tube dependence in patients with primary TLM [25]. Another study assessing quality of life (QOL) in patients treated with TORS showed worse speech, diet, and eating scores at 6 and 12 months in patients with TORS followed by adjuvant RT [26]. Considering the additional toxicities associated with adjuvant therapy, determining an optimal primary treatment method becomes even more important for patients with early-stage hpv+OPC who have a good prognosis and would be expected to survive for a much longer period of time [22]. Furthermore, several previous studies have revealed that primary RT and surgery have different patterns of toxicity. Patients treated with primary RT showed better swallowing function than those treated with surgery, whereas they experienced more hearing loss, mucositis, and tinnitus. Higher incidence of dysphagia, trismus, and postoperative bleeding was also observed in patients treated with primary surgery compared with patients undergoing primary RT [9]. Our study also observed that late grade $\geq 3$ dysphagia, aspiration, and oral cavity bleeding were more frequently reported in the primary surgery group. Regarding the different toxicity profiles of the two treatment methods, management with multimodal adjuvant treatment might make patients vulnerable to all the side effects that increase the risk of late toxicities that interfere with QOL. However, trials to de-intensify RT dose for earlystage hpv+OPC are ongoing [24], making it highly likely that de-escalated RT doses would be established in actual clinical practice in the near future, which might further reduce the RT-induced sequelae. Comprehensively, it would be helpful for the patient to select the primary treatment method considering the similar clinical outcomes of primary surgery and $\mathrm{RT}$, the high rate of adjuvant CCRT after primary surgery, and the possibility of de-intensification of the primary RT dose.

As one of the objectives of this study, we identified preoperative clinical factors that can predict the necessity for postoperative (CC)RT. Some previous studies have analyzed the risk factors for adjuvant therapy based on pathologic 
findings after primary surgery [11-13,27]. In a recently published study, researchers analyzed clinical, pathologic, and socioeconomic predictors of adjuvant therapy in patients with TORS. However, only basic clinical characteristics such as sex, age, and stage were included in the analysis, and any clinical factors that could be helpful in deciding the actual treatment method could not be found [28]. Based on the results of the current study, preoperative clinical findings of LN metastases are predictive and prognostic factors for adjuvant therapy. In particular, it might be better to perform primary (CC)RT in patients with two or more clinically metastatic LNs or ENEcong preoperatively to avoid trimodal treatment in early-stage hpv+OPC.

Our study provided substantial evidence that ENEcong was significantly associated with poor oncologic results. Several studies have shown that ENE is a prognostic factor in hpv+OPC [18,29], although insufficient, it has been reported in one study as a predictive marker of ENEcong for distant metastasis (DM) [21]. First of all, in previous studies, cENE was found to be an independent prognostic factor in cT1-2N1 hpv+OPC [20], and there was a proposal to refine $\mathrm{N}$ classification to include the concept of cENE in hpv+OPC [19]. Although not studied in OPC, conglomerated LNs in laryngeal carcinomas has been found to have a detrimental effect on survival. The five-year survival rate was $70.1 \%$ in the absence of conglomerated LN, but it was reduced to $37.2 \%$ in the presence of conglomerated LNs $(\mathrm{p}=0.025)$ [30]. As previously mentioned, ENEcong has been explored only in one study of patients with advanced-stage III/IV OPC. This study described 'matted nodes' as the imaging characteristics of multiple confluent regional metastases, which was similar to our 'ENEcong'. Of 205 patients, $84 \%$ were HPV-positive, and 3-year disease-specific survival for patients with matted nodes was significantly worse than with nonmatted nodes. Furthermore. the positive predictive value of matted nodes for DM was $66 \%$ [21]. Therefore, although additional relevant studies are lack, ENEcong might be a meaningful indicator to predict adjuvant CCRT in patients with the upfront surgery. Of course, further studies are needed to verify this issue. Another concern is the discrepancy between cENE and ENEcong results as predictors of the need for postoperative treatment. In an analysis of S1 Table, we found out ENEcong was a statistically meaningful indicator to have poor PFS and LC compared to the cENE. And, although no significant difference was reported, 2-year OS was lower when ENEcong existed. These results are believed to have contributed to the difference between the two as predictors, which also requires further researches in large-scale studies.

Some limitations need to be addressed. First, our study has inherently flaws of a retrospective research. Second, a significant number of patients in the primary surgery group under- went surgery with oropharyngectomy rather than TORS/ TLM. Last, our results came from AJCC 8th edition staging system, part of patients before AJCC 8th edition were treated based on National Comprehensive Cancer Network (NCCN) guidelines which were relied on AJCC 7th edition. Thus, the treatment strategies might seem to have any possibility of over-intensification in both two treatments. First of all, although this study was conducted as a multicenter study involving three institutions, it was difficult to have a large number of sample size due to the inclusion of only patients with early-stage hpv+OPC. To address the second limitation, microinvasive surgical techniques have been gradually introduced worldwide since 2010. However, our institutions did not frequently perform TORS/TLM in the early era of introduction of these techniques, and thus oropharyngectomy including tonsillectomy and oropharyngeal excision are still performed when patients' cases are suitable. Regarding the discrepancy of editions of staging system, it was an inevitable issue in this kind of validation study conducted in the transition period of changes in staging systems. However, since the revision of TNM staging, NCCN guideline have been the basis for decisions of all clinicians' treatment policy, and our institution's treatment policy have also been based on them at each applicable time period. Hence, despite the several limitations, our results are clinically meaningful because of the homogenous inclusion of early-stage hpv+ $\mathrm{OPC}$ and the identification of prognostic factors for predicting the postoperative (CC)RT in patients treated with primary surgery. As the management of hpv+OPC needs to be performed by a personalized approach in a multidisciplinary team, our findings might aid physicians in determining which treatment method as tailored to the individual patient. Ongoing prospective randomized trials should clarify the comparison between primary RT and surgery.

\section{Electronic Supplementary Material}

Supplementary materials are available at Cancer Research and Treatment website (https://www.e-crt.org).

\section{Ethical Statement}

After the approval of the Institutional Review Board off the three institutions (No. J-2004-131-1117; B-2005/612-403; 20200327/202020-28/042). Because of the retrospective design of the analysis, the requirement to obtain informed consent of participants included in the study was exempted.

\section{Author Contributions}

Conceived and designed the analysis: Kim DY, Wu HG, Kim JH, Lee JH, Wee CW.

Collected the data: Kim DY, Wu HG, Kim JH, Lee JH, Ahn SH, Chung EJ, Eom KY, Jung YH, Jeong WJ, Kwon TK, Kim S, Wee CW. 
Contributed data or analysis tools: Kim DY, Wu HG, Kim JH, Lee JH, Ahn SH, Chung EJ, Eom KY, Jung YH, Jeong WJ, Kwon TK, Kim $\mathrm{S}$, Wee CW.

Performed the analysis: Kim DY, Wee CW.

Wrote the paper: Kim DY, Wee CW.

\section{Conflicts of Interest}

Conflict of interest relevant to this article was not reported.

\section{References}

1. Baskin RM, Boyce BJ, Amdur R, Mendenhall WM, Hitchcock $\mathrm{K}$, Silver N, et al. Transoral robotic surgery for oropharyngeal cancer: patient selection and special considerations. Cancer Manag Res. 2018;10:839-46.

2. Morisod B, Simon C. Meta-analysis on survival of patients treated with transoral surgery versus radiotherapy for early-stage squamous cell carcinoma of the oropharynx. Head Neck. 2016;38 Suppl 1:E2143-50.

3. Yamashita Y, Ikegami T, Hirakawa H, Uehara T, Deng Z, Agena $S$, et al. Staging and prognosis of oropharyngeal carcinoma according to the 8th edition of the American Joint Committee on Cancer Staging Manual in human papillomavirus infection. Eur Arch Otorhinolaryngol. 2019;276:827-36.

4. Ang KK, Harris J, Wheeler R, Weber R, Rosenthal DI, NguyenTan PF, et al. Human papillomavirus and survival of patients with oropharyngeal cancer. N Engl J Med. 2010;363:24-35.

5. Zanoni DK, Patel SG, Shah JP. Changes in the 8th edition of the American Joint Committee on Cancer (AJCC) staging of head and neck cancer: rationale and implications. Curr Oncol Rep. 2019;21:52.

6. You EL, Henry M, Zeitouni AG. Human papillomavirus-associated oropharyngeal cancer: review of current evidence and management. Curr Oncol. 2019;26:119-23.

7. Gillison ML, Trotti AM, Harris J, Eisbruch A, Harari PM, Adelstein DJ, et al. Radiotherapy plus cetuximab or cisplatin in human papillomavirus-positive oropharyngeal cancer (NRG Oncology RTOG 1016): a randomised, multicentre, noninferiority trial. Lancet. 2019;393:40-50.

8. Fundakowski CE, Lango M. Considerations in surgical versus non-surgical management of HPV positive oropharyngeal cancer. Cancers Head Neck. 2016;1:6.

9. Nichols AC, Theurer J, Prisman E, Read N, Berthelet E, Tran E, et al. Radiotherapy versus transoral robotic surgery and neck dissection for oropharyngeal squamous cell carcinoma (ORATOR): an open-label, phase 2, randomised trial. Lancet Oncol. 2019;20:1349-59.

10. Mirghani H, Blanchard P. Treatment de-escalation for HPVdriven oropharyngeal cancer: where do we stand? Clin Transl Radiat Oncol. 2018;8:4-11.

11. National Comprehensive Cancer Network. Head and neck cancers (version 1.2021) [Internet]. Plymouth Meeting, PA: National Comprehensive Cancer Network; 2021 [cited 2021 Jan 10]. Available from: https://www.nccn.org/professionals/ physician_gls/pdf/head-and-neck.pdf.

12. Cooper JS, Zhang Q, Pajak TF, Forastiere AA, Jacobs J, Saxman
SB, et al. Long-term follow-up of the RTOG 9501/intergroup phase III trial: postoperative concurrent radiation therapy and chemotherapy in high-risk squamous cell carcinoma of the head and neck. Int J Radiat Oncol Biol Phys. 2012;84:1198205.

13. Geiger JL, Ku JA. Postoperative treatment of oropharyngeal cancer in the era of human papillomavirus. Curr Treat Options Oncol. 2019;20:20.

14. Mehanna H, Robinson M, Hartley A, Kong A, Foran B, Fulton-Lieuw $\mathrm{T}$, et al. Radiotherapy plus cisplatin or cetuximab in low-risk human papillomavirus-positive oropharyngeal cancer (De-ESCALaTE HPV): an open-label randomised controlled phase 3 trial. Lancet. 2019;393:51-60.

15. Peng H, Chen L, Tang LL, Li WF, Mao YP, Guo R, et al. Significant value of (18)F-FDG-PET/CT in diagnosing small cervical lymph node metastases in patients with nasopharyngeal carcinoma treated with intensity-modulated radiotherapy. Chin J Cancer. 2017;36:95.

16. Biau J, Lapeyre M, Troussier I, Budach W, Giralt J, Grau C, et al. Selection of lymph node target volumes for definitive head and neck radiation therapy: a 2019 update. Radiother Oncol. 2019;134:1-9.

17. Billfalk-Kelly A, Yu E, Su J, O'Sullivan B, Waldron J, Ringash J, et al. Radiologic extranodal extension portends worse outcome in $\mathrm{cN}+\mathrm{TNM}-8$ stage I human papillomavirusmediated oropharyngeal cancer. Int J Radiat Oncol Biol Phys. 2019;104:1017-27.

18. Freitag J, Wald T, Kuhnt T, Gradistanac T, Kolb M, Dietz A, et al. Extracapsular extension of neck nodes and absence of human papillomavirus 16-DNA are predictors of impaired survival in p16-positive oropharyngeal squamous cell carcinoma. Cancer. 2020;126:1856-72.

19. Huang SH, O'Sullivan B, Su J, Bartlett E, Kim J, Waldron JN, et al. Prognostic importance of radiologic extranodal extension in HPV-positive oropharyngeal carcinoma and its potential role in refining TNM-8 cN-classification. Radiother Oncol. 2020;144:13-22.

20. Lee HN, Han JK, Kim HH, Shin HC, Kim IY, Jou SS. Comparative study of lymph node metastasis from squamous cell carcinoma and non-squamous cell carcinoma on neck CT. J Korean Soc Radiol. 2015;72:271-81.

21. Spector ME, Chinn SB, Bellile E, Gallagher KK, Ibrahim M, Vainshtein $\mathrm{J}$, et al. Matted nodes as a predictor of distant metastasis in advanced-stage III/IV oropharyngeal squamous cell carcinoma. Head Neck. 2016;38:184-90. 
22. Stelmes JJ, Gregoire V, Poorten VV, Golusinski W, Szewczyk $\mathrm{M}$, Jones $\mathrm{T}$, et al. Organ preservation and late functional outcome in oropharyngeal carcinoma: rationale of EORTC 1420, the "Best of" trial. Front Oncol. 2019;9:999.

23. Nichols AC, Lang P, Prisman E, Berthelet E, Tran E, Hamilton $S$, et al. Treatment de-escalation for HPV-associated oropharyngeal squamous cell carcinoma with radiotherapy vs. trans-oral surgery (ORATOR2): study protocol for a randomized phase II trial. BMC Cancer. 2020;20:125.

24. Yom SS, Torres-Saavedra P, Caudell JJ, Waldron JN, Gillison $\mathrm{ML}$, Xia P, et al. Reduced-dose radiation therapy for HPVassociated oropharyngeal carcinoma (NRG Oncology HN002). J Clin Oncol. 2021;39:956-65.

25. Haughey BH, Hinni ML, Salassa JR, Hayden RE, Grant DG, Rich JT, et al. Transoral laser microsurgery as primary treatment for advanced-stage oropharyngeal cancer: a United States multicenter study. Head Neck. 2011;33:1683-94.

26. Leonhardt FD, Quon H, Abrahao M, O'Malley BW Jr, Weinstein GS. Transoral robotic surgery for oropharyngeal carcinoma and its impact on patient-reported quality of life and function. Head Neck. 2012;34:146-54.

27. Langendijk JA, Slotman BJ, van der Waal I, Doornaert P, Berkof J, Leemans CR. Risk-group definition by recursive partitioning analysis of patients with squamous cell head and neck carcinoma treated with surgery and postoperative radiotherapy. Cancer. 2005;104:1408-17.

28. Baliga S, Klamer B, Jhawar S, Gamez M, Mitchell D, Blakaj A, et al. Identification of clinical and socioeconomic predictors of adjuvant therapy after trans-oral robotic surgery in patientswithoropharyngealsquamouscellcarcinoma.Cancers (Basel). 2020;12:2474.

29. Lee B, Choi YJ, Kim SO, Lee YS, Hong JY, Baek JH, et al. Prognostic value of radiologic extranodal extension in human papillomavirus-related oropharyngeal squamous cell carcinoma. Korean J Radiol. 2019;20:1266-74.

30. Ozmen OA, Alpay M, Saraydaroglu O, Demir UL, Kasapoglu F, Coskun HH, et al. Prognostic significance of soft tissue deposits in laryngeal carcinoma. Braz J Otorhinolaryngol. 2018;84:566-73. 